\title{
INTERSECTION NUMBERS OF EXTREMAL RAYS ON HOLOMORPHIC SYMPLECTIC VARIETIES*
}

\author{
BRENDAN HASSETT ${ }^{\dagger}$ AND YURI TSCHINKEL ${ }^{\ddagger}$
}

Key words. Holomorphic symplectic varietes, Beauville-Bogomolov form, extremal rays.

AMS subject classifications. Primary 14E30; Secondary 14C20, 14 J40

1. Introduction. Suppose $X$ is a smooth projective complex variety. Let $\mathrm{N}_{1}(X, \mathbb{Z}) \subset \mathrm{H}_{2}(X, \mathbb{Z})$ and $\mathrm{N}^{1}(X, \mathbb{Z}) \subset \mathrm{H}^{2}(X, \mathbb{Z})$ denote the group of curve classes modulo homological equivalence and the Néron-Severi group respectively. The monoids of effective classes in each group generate cones $\mathrm{NE}_{1}(X) \subset \mathrm{N}_{1}(X, \mathbb{R})$ and $\mathrm{NE}^{1}(X) \subset \mathrm{N}^{1}(X, \mathbb{R})$ with closures $\overline{\mathrm{NE}}_{1}(X)$ and $\overline{\mathrm{NE}}^{1}(X)$, the pseudoeffective cones. These play a central rôle in the birational geometry of $X$. By Kleiman's criterion [17], a divisor $D$ is ample if and only if $D \cdot C>0$ for each $C \in \overline{\mathrm{NE}}_{1}(X) \backslash\{0\}$. More generally, a divisor $D$ is nef if $D \cdot C \geq 0$ for each such $C$. Divisors in the interior of $\mathrm{NE}^{1}(X)$ are big and induce birational maps from $X$. In general, very little is known about the structure of these cones beyond Mori's Cone Theorem [5, §3.3] and more general finiteness results arising from the Minimal Model Program [2, 1.1.9].

Let $X$ be an irreducible holomorphic symplectic variety, i.e., a smooth projective simply-connected manifold admitting a unique nondegenerate holomorphic two-form. Let $($,$) denote the Beauville-Bogomolov form on the cohomology group \mathrm{H}^{2}(X, \mathbb{Z})$, normalized so that it is integral and primitive. Duality gives a $\mathbb{Q}$-valued form on $\mathrm{H}_{2}(X, \mathbb{Z})$, also denoted $($,$) . When X$ is a $\mathrm{K} 3$ surface these coincide with the intersection form.

For a polarized K3 surface $(S, g)$, we can read off the ample cone from the Hodge structure on the middle cohomology and the polarizing class. Precisely, the cone of effective curves can be expressed [18]

$$
\mathrm{NE}_{1}(S)=\left\langle C \in \mathrm{N}_{1}(S, \mathbb{Z}):(C, C) \geq-2, C \cdot g>0\right\rangle .
$$

(We use the notation $\langle\cdots\rangle$ for the 'cone generated by the enclosed elements'.) Thus the ample cone can be expressed

$$
\left\langle h \in \mathrm{N}^{1}(S, \mathbb{Z}): h \cdot C>0, \forall C \in \mathrm{N}_{1}(S, \mathbb{Z}) \text { with }(C, C) \geq-2, C \cdot g>0\right\rangle .
$$

Our goal is to extend these explicit descriptions to higher dimensions. Of course, on surfaces curves and divisors are equivalent. In higher dimensions we can seek characterizations of both the effective curves and the effective divisors.

Thesis 1.1. Let $X$ be an irreducible holomorphic symplectic manifold of dimension $2 n$. There is a universal constant $c_{X} \in \mathbb{Q}_{>0}$ depending only on the deformation equivalence class of $X$, with the following properties:

- Let $X^{\prime}$ be a deformation of $X$ containing a Lagrangian submanifold $P^{\prime} \subset X^{\prime}$ with $P^{\prime} \simeq \mathbb{P}^{n}$. Let $\ell$ denote the class of a line on $P^{\prime}$ interpreted as an element of $\mathrm{H}_{2}(X, \mathbb{Z}) \subset \mathrm{H}^{2}(X, \mathbb{Z}) \otimes_{\mathbb{Z}} \mathbb{Q}$. Then $(\ell, \ell)=-c_{X}$.

\footnotetext{
${ }^{*}$ Received March 18, 2010; accepted for publication April 23, 2010.

${ }^{\dagger}$ Department of Mathematics, Rice University, 6100 S. Main St., Houston, TX 77251, USA (has sett@rice.edu).

$\ddagger$ Courant Institute of Mathematical Sciences, New York University, 251 Mercer St., New York, NY 10012, USA (tschinkel@cims.nyu.edu).
} 
- Assume that $X$ admits a polarization $g$. Then we have

$$
\mathrm{NE}_{1}(X)=\left\langle C \in \mathrm{N}_{1}(X, \mathbb{Z}):(C, C) \geq-c_{X}, C \cdot g>0\right\rangle .
$$

Our main objective is to collect evidence supporting this thesis for various deformation equivalence classes of irreducible holomorphic symplectic manifolds. In doing so, we formulate precise conjectures in these cases.

It is natural to seek to relate the intersection properties of extremal rays $R \in$ $\mathrm{N}_{1}(X, \mathbb{Z})$ to the geometry of the associated contractions. We carry out this analysis for divisorial contractions in Section 2, and obtain new structural results on cones of effective divisors.

Conventions. Let $R \in \mathrm{N}_{1}(X, \mathbb{Z})$ denote the primitive generator of an extremal ray on $\overline{\mathrm{NE}}_{1}(X)$ with negative self-intersection. Let $\rho \in \mathrm{N}^{1}(X, \mathbb{Z})$ denote the divisor class corresponding to $\rho$, i.e., using the form (, ) to embed

$$
\mathrm{H}^{2}(X, \mathbb{Z}) \subset \mathrm{H}_{2}(X, \mathbb{Z})
$$

we take $\rho$ to be the smallest positive multiple of $R$ contained in $\mathrm{H}^{2}(X, \mathbb{Z})$.

The notation $S \sim M$ means ' $S$ is deformation equivalent to $M$ '.

Punctual Hilbert schemes. Let $X$ be deformation equivalent to the punctual Hilbert scheme $S^{[n]}$, where $S$ is a K3 surface. For $n>1$ the Beauville-Bogomolov form can be written $[1, \S 8]$

$$
\mathrm{H}^{2}(X, \mathbb{Z}) \simeq \mathrm{H}^{2}(S, \mathbb{Z})_{(,)} \oplus_{\perp} \mathbb{Z} \delta, \quad(\delta, \delta)=-2(n-1)
$$

where $2 \delta$ is the class of the 'diagonal' divisor $\Delta^{[n]} \subset S^{[n]}$ parametrizing non-reduced subschemes. For each homology class $f \in \mathrm{H}^{2}(S, \mathbb{Z})$, let $f \in \mathrm{H}^{2}(X, \mathbb{Z})$ denote the class parameterizing subschemes with some support along $f$. This is compatible with the lattice embedding above. Duality gives a $\mathbb{Q}$-valued form on homology

$$
\mathrm{H}_{2}(X, \mathbb{Z}) \simeq \mathrm{H}_{2}(S, \mathbb{Z})_{(,)} \oplus_{\perp} \mathbb{Z} \delta^{\vee}, \quad\left(\delta^{\vee}, \delta^{\vee}\right)=-1 / 2(n-1)
$$

where $\delta^{\vee}$ is characterized as the homology class orthogonal to $\mathrm{H}^{2}(S, \mathbb{Z})$ and satisfying $\delta^{\vee} \cdot \delta=1$.

Our thesis takes the following form:

Conjecture 1.2. Let $(X, g)$ be a polarized variety deformation equivalent to $S^{[n]}$ where $S$ is a K3 surface. Then

$$
\mathrm{NE}_{1}(X)=\left\langle R \in \mathrm{N}_{1}(X, \mathbb{Z}):(R, R) \geq-(n+3) / 2, R \cdot g>0\right\rangle
$$

and a divisor class $h$ on $X$ is ample if $h \cdot R>0$ for each $R \in \mathrm{N}_{1}(X, \mathbb{Z})$ satisfying $g \cdot R>0$ and

$$
(R, R) \geq-(n+3) / 2
$$

This generalizes to higher dimensions conjectures of [8] in the special case $n=2$; see [9] for a proof in this case that the divisors predicted to be nef are in fact nef.

For the examples in dimensions $\leq 8$ discussed below, the geometry of each extremal ray is characterized by its intersection properties: Let $R$ be a primitive integral 
class with $(R, R)<0$ generating an extremal ray of the cone $\overline{\mathrm{NE}}_{1}(X)$. Choose $k \in \mathbb{N}$ such that

$$
-(k+3) / 2 \leq(R, R)< \begin{cases}-(k+2) / 2 & \text { if } k>1 \\ 0 & \text { if } k=1 .\end{cases}
$$

Then there exists an extremal contraction $\beta: X \rightarrow Y$ of $R$

$$
\begin{array}{llllll}
\mathbb{P}^{k} \rightarrow & Z_{\circ} & \subset & Z & \subset & X \\
& \beta_{\circ} \downarrow & & \downarrow & & \beta \downarrow \\
& W_{\circ} & \subset & W & \subset & Y
\end{array}
$$

where $Y$ is the image, $Z$ the exceptional locus, $W$ its image, and $W_{\circ} \subset W$ is an open subset over which $\beta_{\circ}$ is smooth. Furthermore, $\beta_{\circ}$ is a $\mathbb{P}^{k}$-bundle over $W_{\circ}$. Theorem 2.3, for general holomorphic symplectic manifolds, shows that divisorial extremal rays satisfy $(R, R) \geq-2$.

It is a general property of birational contractions of holomorphic symplectic manifolds [16, Theorem 2.3] (cf. Namikawa [26]) that $W_{\circ}$ is holomorphic symplectic and $W \backslash W_{\circ}$ has even codimension in $Y$. Kaledin shows more: He gives a finite 'Poisson stratification' of $W$.

REMARK 1.3. For $n=5$, the geometric characterization sketched above is incompatible with Conjecture 1.2. There are elements $R \in \mathrm{H}_{2}\left(S^{[5]}, \mathbb{Z}\right)$ with $(R, R)=-9 / 8$; however, a result of Markman implies these cannot correspond to divisorial contractions. See Remark 2.5 for details.

Finally, the results of $[8, \S 4]$ (building on [27]) imply that these conjectures are stable under small deformations: Given a negative extremal ray $R=[\ell]$ for $X$ with an interpretation as above, for any small deformation $X^{\prime}$ of $X$ such that the class $R$ remains algebraic, there exists an $\ell^{\prime} \subset X^{\prime}$ specializing to $\ell$ and subject to the same geometric interpretation.

Generalized Kummer varieties. Suppose that $X$ is a holomorphic symplectic variety of dimension $2 n$, deformation equivalent to a generalized Kummer variety $K_{n}(A)$, defined as follows: Given an abelian surface $A$ with degree $(n+1)$ Hilbert scheme $A^{[n+1]}, K_{n}(A)$ is the fiber over 0 of the addition map $\alpha: A^{[n+1]} \rightarrow A$. Here the Beauville-Bogomolov form is

$$
\mathrm{H}^{2}\left(K_{n}(A), \mathbb{Z}\right)=\mathrm{H}^{2}(A, \mathbb{Z}) \oplus_{\perp} \mathbb{Z} e, \quad(e, e)=-2(n+1)
$$

where $2 e$ is the class of the non-reduced subschemes [32, §4.3.1]. (Each class $\eta \in$ $\mathrm{H}^{2}(A, \mathbb{Z})$ yields a class in $\mathrm{H}^{2}\left(K_{n}(A), \mathbb{Z}\right)$, i.e., the subschemes with some support along $\eta$.) As in the Hilbert scheme case, we use (, ) to embed $\mathrm{H}^{2}(X, \mathbb{Z}) \subset \mathrm{H}_{2}(X, \mathbb{Z})$ and extend $($,$) to a \mathbb{Q}$-valued form on $\mathrm{H}_{2}(X, \mathbb{Z})$.

We offer a precise statement analogous to the conjectures in [8] for varieties deformation equivalent to $S^{[2]}$ :

Conjecture 1.4. Let $(X, g)$ be a polarized variety deformation equivalent to a four-dimensional generalized Kummer variety. Then

$$
\mathrm{NE}_{1}(X)=\left\langle R \in \mathrm{N}_{1}(X, \mathbb{Z}):(R, R) \geq-3 / 2, R \cdot g>0\right\rangle
$$

and a divisor class $h$ on $X$ is ample if and only if $h \cdot R>0$ for each $R \in \mathrm{N}_{1}(X, \mathbb{Z})$ satisfying $g \cdot R>0$ and

$$
(R, R) \geq-3 / 2 \text {. }
$$


Furthermore, extremal rays $R$ with $(R, R)<0$ have the following interpretations:

- $(R, R)=-\frac{1}{6}$ : There exists a divisor $E \subset X$ with class $[E]=2 e$ where $e=6 R$, with $E$ ruled over an abelian surface with fibers of class $R$.

- $(R, R)=-\frac{2}{3}$ : There exists a divisor $F \subset X$ where $F=3 R$, with $F$ ruled over an abelian surface with fibers of class $R$.

- $(R, R)=-\frac{3}{2}$ : There exists a plane $P \subset X$ whose lines $\ell \subset P$ have class $R$.

In particular, if $\rho$ is the smallest positive multiple of $R$ contained in $\mathrm{H}^{2}(X, \mathbb{Z})$ (via the inclusion $\left.\mathrm{H}^{2}(X, \mathbb{Z}) \subset \mathrm{H}_{2}(X, \mathbb{Z})\right)$ then in each case

$$
(\rho, \rho)=(e, e)=-6 .
$$

In [11], we prove this last statement when $X$ contains a Lagrangian plane $P$ and $\rho$ is associated with the class of a line $\ell \subset P$; if $R=[\ell]$ is primitive then $(R, R)=-\frac{3}{2}$.

Acknowledgments. We are grateful to L. Göttsche for useful conversations. We are grateful to D. Kaledin for reading an early draft of this manuscript, and to E. Markman for pointing out the results of Boucksom and Druel we utilize. The first author was supported by National Science Foundation Grants 0554491 and 0901645; the second author was supported by National Science Foundation Grants 0554280 and 0602333.

2. Divisorial contractions. Let $X$ be an irreducible holomorphic symplectic manifold of dimension $2 n$. What is needed to prove that the divisors predicted to be ample are indeed ample? This would entail showing that all negative extremal rays $R$ on $X$ satisfy $(R, R) \geq-c_{X}$. In this section, we establish some uniform bounds of this type, and offer applications to cones of moving and effective divisors.

Let $\beta: X \rightarrow Y$ be an extremal contraction of the ray $R$. Let $Y_{\text {sing }}$ denote the singular locus of $Y$; it is a result of Namikawa $[26,1.4]$ that $Y_{\text {sing }}$ is even dimensional. Moreover, Kaledin $[16,2.3]$ has shown that $Y_{\text {sing }}$ is stratified by symplectic manifolds, and $\beta$ has fiber dimension $n-j$ over the $(2 j)$-dimensional stratum.

Thus $\beta$ is divisorial if and only if $Y_{\text {sing }}$ has codimension two. In this situation [26, $\S 1]$ and $[29,5.1]$ offer detailed structural information about the singularities at the generic point of $Y_{\text {sing }}$, i.e., they are rational double points of types $A_{1}$ or $A_{2}$. Let $E$ be the exceptional divisor and $C$ the class of an exceptional rational curve. Assume that $C$ is the class of an irreducible component $\ell$ of the fiber of $\beta$ over a generic point of $Y_{\text {sing. }}$. First, we have

$$
E \cdot C= \begin{cases}-2 & \text { in the } A_{1} \text { case } \\ -1 & \text { in the } A_{2} \text { case. }\end{cases}
$$

Regard the classes of $E$ and $C \in \mathrm{H}_{2}(X, \mathbb{Q})=\mathrm{H}^{2}(X, \mathbb{Q})$; the class of $E$ is a positive rational multiple of $C$. Indeed, we may deform $X$ preserving the algebraicity of the class of $C$; the curve $C$ deforms to a curve in nearby fibers [8, §4], which still sweeps out a divisor that specializes back to $E$.

Express $E=m \rho$ and $C=m^{\prime} R$ for $\rho \in \mathrm{H}^{2}(X, \mathbb{Z})$ and $R \in \mathrm{H}_{2}(X, \mathbb{Z})$ primitive and $m, m^{\prime} \in \mathbb{N}$. We have $\rho=k R$ for some $k \in \mathbb{N}$ as well, since the Beauville-Bogomolov form induces an inclusion $\mathrm{H}^{2}(X, \mathbb{Z}) \subset \mathrm{H}_{2}(X, \mathbb{Z})$. Thus we have

$$
E \cdot C=m m^{\prime} \rho \cdot R=m m^{\prime} k(R, R),
$$

which implies that $(R, R) \geq-2$. 
We summarize this discussion in the following theorem:

TheOREm 2.1. Let $X$ be an irreducible holomorphic symplectic manifold. Let $R$ be a primitive generator of an extremal ray of $X$ such that the associated extremal contraction is divisorial. Then

$$
-2 \leq(R, R)<0 .
$$

REMARK 2.2. Our argument works under slightly weaker assumptions: It suffices that $\beta: X \rightarrow Y$ be a divisorial contraction with irreducible exceptional divisor $E$, and that $R$ be the primitive class associated with an irreducible component of the generic exceptional fiber. Since $E$ is irreducible, the monodromy action on the irreducible components of the generic exceptional fiber is transitive. This suffices to show that $Y$ has $A_{1}$ or $A_{2}$ singularities at the generic point of $Y_{\text {sing }}$.

TheOREM 2.3. Let $(X, g)$ be a polarized irreducible holomorphic symplectic manifold. The cone of effective divisors $\operatorname{NE}^{1}(X)$ satisfies

$$
\langle R:(R, R)>0, R \cdot g>0\rangle \subset \mathrm{NE}^{1}(X) \subset\langle R:(R, R) \geq-2, R \cdot g>0\rangle,
$$

where $R$ is taken in $\mathrm{N}_{1}(X, \mathbb{Z})$.

Note that the cones naturally sit in $\mathrm{N}_{1}(X, \mathbb{R})$. However, the Beauville-Bogomolov form allows us to identify $\mathrm{N}_{1}(X, \mathbb{R})=\mathrm{N}^{1}(X, \mathbb{R})$.

Proof. If $D \in \mathrm{N}^{1}(X, \mathbb{Z})$ satisfies $(D, D)>0$ then $D$ or $-D$ is big $[12,3.10]$ and thus in the effective cone. It remains to understand the effective divisors $D$ with $(D, D)<0$. (The existence of a non-trivial divisor $D$ on $X$ with $(D, D)=0$ is conjectured to imply that $X$ is birational to an abelian fibration [8, Conj. 3.8] [28, Conj. 4.2].)

We are grateful to E. Markman for drawing our attention to the following result, which combines [6, Prop. 1.4] and [3, Thm. 4.5]:

Let $(X, g)$ be a polarized irreducible holomorphic symplectic manifold. Suppose that $E$ is an irreducible divisor on $X$ with $(E, E)<0$. Then there exists a smooth irreducible holomorphic symplectic variety $X^{\prime}$ birational to $X$ such that the corresponding divisor $E^{\prime} \subset X^{\prime}$ is contractible.

Thus our analysis of extremal contractions applies to $E^{\prime} \subset X^{\prime}$; we can use the birational map to translate this information back to $X$. Indeed, this map gives a natural identification $\mathrm{H}^{2}(X, \mathbb{Z})=\mathrm{H}^{2}\left(X^{\prime}, \mathbb{Z}\right)$ preserving the Beauville-Bogomolov form; by duality, there is an induced identification $\mathrm{H}_{2}(X, \mathbb{Z})=\mathrm{H}_{2}\left(X^{\prime}, \mathbb{Z}\right)$.

The analysis of Theorem 2.1 (extended via Remark 2.2) shows that there exists a class $R^{\prime} \in \mathrm{N}_{1}\left(X^{\prime}, \mathbb{Z}\right)$ proportional to $E^{\prime}$ such that $\left(R^{\prime}, R^{\prime}\right) \geq-2$. Now $E$ and $E^{\prime}$ are identified, as are $R$ and $R^{\prime}$. In particular, $E$ is proportional to a class $R \in \mathrm{N}_{1}(X, \mathbb{Z})$ with $(R, R) \geq-2$. $\mathrm{\square}$

Corollary 2.4. Let $M$ be a divisor class such that $M \cdot R \geq 0$ for each $R \in$ $\mathrm{N}_{1}(X, \mathbb{Z})$ with $R \cdot g>0$ and $(R, R) \geq-2$. Then $M$ is in the closure of the moving cone.

The closure of the moving cone coincides with the intersection of the closure of the birational Kähler cone with $\mathrm{N}^{1}(X, \mathbb{R})[9$, Thm. 7, Cor. 19]. By [13, Prop. 4.2], a 
divisor $M$ is in the closure of the birational Kähler cone if $(M, D) \geq 0$ for all uniruled divisors $D$. This condition follows from Theorem 2.3.

REMARK 2.5. Markman [21, Thm. 1.2] establishes further intersection-theoretic properties of exceptional divisors on varieties $X$ deformation equivalent to length $n \geq 2$ Hilbert schemes of K3 surfaces. His approach is to classify possible 'PicardLefschetz' type reflections on the cohomology lattice. If $\rho$ is a primitive divisor class associated to such a divisor then

$$
(\rho, \rho)=-2,-2(n-1) .
$$

In the latter case, $\rho$ is divisible by $(n-1)$ in $\mathrm{H}_{2}(X, \mathbb{Z})$, i.e.,

$$
\rho=(n-1) m R
$$

for some positive integer $m$. Thus we have

$$
(R, R)=-2,-\frac{2}{m^{2}(n-1)} .
$$

3. Data for Hilbert schemes of K3 surfaces. In this section, we tabulate data for extremal rays with prescribed intersection properties, with references to explicit varieties exhibiting each type. We focus on $S^{[n]}$ for $n \leq 4$. Our objective is to provide evidence for the conjectures in the introduction.

\begin{tabular}{|c|c|l|}
\hline$(R, R)$ & $(\rho, \rho)$ & Geometry \\
\hline-2 & -2 & $\mathbb{P}^{1}$ \\
\hline
\end{tabular}

TABLE H1. $X \sim S$

\begin{tabular}{|c|c|l|l|}
\hline$(R, R)$ & $(\rho, \rho)$ & Geometry & Example \\
\hline$-\frac{1}{2}$ & -2 & Diagonal $\mathbb{P}^{1}$-bundle over $M \sim S$ & $4.3,4.2$ \\
-2 & -2 & $\mathbb{P}^{1}$-bundle over $M \sim S$ & 4.7 \\
$-\frac{5}{2}$ & -10 & $\mathbb{P}^{2}$ (Lagrangian) & $4.12,4.11$ \\
\hline
\end{tabular}

TABle H2. $X \sim S^{[2]}$

See [8] for additional representative examples. In [9] we proved that every extremal ray with negative self-intersection fits into one of these three classes. In particular, the divisors asserted to be ample in Conjecture 1.2 are indeed ample. In [10] we proved the Conjecture 1.2 in a specific example, i.e., the variety of lines on a cubic fourfold admitting a hyperplane section with six double points. 


\begin{tabular}{|c|c|l|l|}
\hline$(R, R)$ & $(\rho, \rho)$ & Geometry & Example \\
\hline$-\frac{1}{4}$ & -4 & $\begin{array}{l}\text { Diagonal } \mathbb{P}^{1} \text {-bundle over } \\
(S \times S)_{\circ}\end{array}$ & 4.2 \\
-2 & -2 & $\mathbb{P}^{1}$-bundle over $M \sim S_{\circ}^{[2]}$ & $4.6(n=3$ case $), 4.7$ \\
$-\frac{9}{4}$ & -36 & $\mathbb{P}^{2}$-bundle over $M \sim S$ & 4.9 \\
-3 & -12 & $\mathbb{P}^{3}$ (Lagrangian) & 4.11 \\
\hline
\end{tabular}

TABLE H3. $X \sim S^{[3]}$

\begin{tabular}{|c|c|l|l|}
\hline$(R, R)$ & $(\rho, \rho)$ & Geometry & Example \\
\hline$-\frac{1}{6}$ & -6 & Diagonal $\mathbb{P}^{1}$-bundle over $M \sim\left(S \times S^{[2]}\right)_{\circ}$ & 4.2 \\
$-\frac{2}{3}$ & -6 & $\begin{array}{l}\mathbb{P}^{1} \text {-bundle over } M \sim\left(S^{[2]} \times S^{\prime}\right)_{\circ}, \\
\text { where } S \text { and } S^{\prime} \text { are isogenous }\end{array}$ & 4.5 \\
-2 & -2 & $\mathbb{P}^{1}$-bundle over $S^{[3]}$ & 4.7 \\
$-\frac{13}{6}$ & -78 & $\mathbb{P}^{2}$-bundle over $S^{[2]}$ & 4.8 \\
$-\frac{8}{3}$ & -24 & $\mathbb{P}^{3}$-bundle over $S$ & 4.10 \\
$-\frac{7}{2}$ & -14 & $\mathbb{P}^{4}$ (Lagrangian) & 4.11 \\
\hline
\end{tabular}

TABLE H4. $X \sim S^{[4]}$

REMARK 3.1. It is natural to ask whether Grassmannians (and Grassmann bundles) also arise as we deform extremal rays in holomorphic symplectic manifolds. There are obvious examples of embeddings

$$
\operatorname{Gr}(m, n) \hookrightarrow X .
$$

Concretely, let $S \subset \mathbb{P}^{3}$ be a quartic K3 surface not containing a line with polarization $f, \operatorname{Gr}(2,4)$ the Grassmannian of lines in $\mathbb{P}^{3}$, and

$$
\begin{aligned}
\operatorname{Gr}(2,4) & \rightarrow S^{[4]} \\
L & \mapsto L \cap S .
\end{aligned}
$$

However, the minimal rational curves in $\operatorname{Gr}(2,4)$ correspond to lines containing a point and contained in a plane. These have class $f-\delta$ in $\mathrm{H}_{2}\left(S^{[4]}, \mathbb{Z}\right)$. But these are not the only extremal rational curves on $S^{[4]}$. Consider general 4 -tuples of points on $S$ that are coplanar. These form a $\mathbb{P}^{1}$-bundle over the relative Jacobian of the linear series $|f|$ on $S$. They also have class $f-\delta$.

There are conceptual reasons why Lagrangian submanifolds swept out by extremal rays are projective spaces and not more general varieties. If $\ell \simeq \mathbb{P}^{1} \subset X$ sweeps out 
a Lagrangian manifold $G$ then

$$
\operatorname{Def}(\ell, G)=\operatorname{Def}(\ell, X) .
$$

In particular, $\Gamma\left(\Omega_{G}^{1} \mid \ell\right)=0$ thus Riemann-Roch implies

$$
\chi\left(\Omega_{G}^{1} \mid \ell\right)=\operatorname{deg}\left(\Omega_{G}^{1} \mid \ell\right)+\operatorname{dim}(G)+1 \leq 0 .
$$

However, this forces $G \simeq \mathbb{P}^{\operatorname{dim}(G)}$; see [4], which also includes classification results for isolated symplectic singularities along these lines.

4. Examples of extremal rays for Hilbert schemes. In general, we do not have a conceptual explanation of the intersection-theoretic properties of negative extremal rays. We therefore give examples for each type enumerated on the table. The monodromy analysis of Markman [19, 20] and Gritsenko-Hulek-Sankaran [7] show that orbits of primitive elements $\rho \in \mathrm{H}^{2}(X, \mathbb{Z})$ under the monodromy representation are governed by intersection-theoretic properties, e.g., by the square $(\rho, \rho)$ and the ideal $\left(\rho, \mathrm{H}^{2}(X, \mathbb{Z})\right) \subset \mathbb{Z}$.

Furthermore, the deformation-theoretic results of $[8,27]$ imply that the examples produced here are stable under deformations preserving the Hodge class of $R$, the negative extremal ray under consideration.

Review of Fourier-Mukai formalism. Let $(S, f)$ be a complex polarized K3 surface. The cohomology

$$
\mathrm{H}^{*}(S, \mathbb{Z})=\mathrm{H}^{0}(S, \mathbb{Z}) \oplus \mathrm{H}^{2}(S, \mathbb{Z}) \oplus \mathrm{H}^{4}(S, \mathbb{Z})
$$

admits a Mukai pairing

$$
\left\langle\left(r_{1}, D_{1}, s_{1}\right),\left(r_{2}, D_{2}, s_{2}\right)\right\rangle=D_{1} \cdot D_{2}-r_{1} s_{2}-r_{2} s_{1} .
$$

This is a unimodular integral quadratic form. We regard the full cohomology ring as a Hodge structure of weight two, with the degree zero and four parts interpreted as Tate Hodge structures $\mathbb{Z}(-1)$.

Let $\mathcal{E}$ be a coherent sheaf on $S$. Its Mukai vector is defined

$$
v(\mathcal{E})=\left(r(\mathcal{E}), c_{1}(\mathcal{E}), s(\mathcal{E}) p\right) \in \mathrm{H}^{*}(S, \mathbb{Z})
$$

where $r(\mathcal{E})$ is the rank, $p$ is the class of a point on $S$, and

$$
s(\mathcal{E})=\chi(S, \mathcal{E})-r(\mathcal{E})=c_{1}(\mathcal{E})^{2} / 2-c_{2}(\mathcal{E})+r(\mathcal{E}) .
$$

Given two coherent sheaves $\mathcal{E}$ and $\mathcal{F}$ and $S$, we have [24, 2.2]

$$
\chi(\mathcal{E}, \mathcal{F}):=\operatorname{Hom}(\mathcal{E}, \mathcal{F})-\operatorname{Ext}^{1}(\mathcal{E}, \mathcal{F})+\operatorname{Ext}^{2}(\mathcal{E}, \mathcal{F})=-\langle v(\mathcal{E}), v(\mathcal{F})\rangle .
$$

A coherent sheaf $\mathcal{E}$ is simple if its only endomorphisms are homotheties, i.e., $\operatorname{Hom}(\mathcal{E}, \mathcal{E})=\mathbb{C}$. Mukai $[22]$ has shown that the moduli space of such sheaves is smooth and holomorphic symplectic of dimension

$$
2-\chi(\mathcal{E}, \mathcal{E})=\langle v(\mathcal{E}), v(\mathcal{E})\rangle .
$$

TheOREm 4.1. Let $(S, f)$ be a general polarized K3 surface of degree $f \cdot f$. Let $v$ be a Mukai vector of a simple sheaf on $S$ and $M_{v}$ the corresponding moduli space of simple 
sheaves. Assume that $v$ is primitive and isotropic with respect to the Mukai pairing. Then each connected component $M \subset M_{v}$ is a K3 surface with period computed by the following identification of Hodge structures

$$
\mathrm{H}^{2}(M, \mathbb{Z})=v^{\perp} / \mathbb{Z} v
$$

Proof. First assume that $(S, f)$ is an arbitrary polarized K3 surface of this degree. Given a polarization $A$ on $S$, we can consider the moduli space $M_{A, v}$ of $A$-stable sheaves with Mukai vector $v$. This is compact provided the numbers $r(\mathcal{E}), s(\mathcal{E})$, and $A \cdot c_{1}(\mathcal{E})$ are relatively prime $[24,4.1]$. Since stable sheaves are automatically simple, the moduli space of simple sheaves is also compact.

Provided $v$ is primitive, we can always specialize $(S, f)$ to obtain an extra polarization $A$ such that $M_{A, v}$ is compact. It follows that $M_{v}$ is also compact, and this remains true after deforming back to a generic $S$.

The computation of the period of $M$ is [24, Thm. 1.4].

Let $\mathcal{E} \rightarrow S \times M$ denote a universal sheaf; the Fourier-Mukai transform of bounded derived categories is defined $[24,1.5]$

$$
\begin{aligned}
\Phi_{\mathcal{E}}: D^{b}(S) & \rightarrow D^{b}(M) \\
\mathcal{F} & \mapsto\left(\pi_{M}\right)_{*}\left(\pi_{S}^{*} \mathcal{F} \otimes_{\mathbb{L}} \mathcal{E}\right),
\end{aligned}
$$

where we take the derived push-forward, pull-back, and tensor-product. Let $\phi_{\mathcal{E}}$ denote the induced map on cohomology, characterized by the formula [14, 5.29]:

$$
\phi_{\mathcal{E}}(\operatorname{ch}(\mathcal{F}) \cdot \sqrt{\operatorname{td}(S)})=\operatorname{ch}\left(\Phi_{\mathcal{E}}(\mathcal{F})\right) \cdot \sqrt{\operatorname{td}(M)}
$$

where ch denotes the Chern character and td the Todd class. Examples of concrete computations in special cases can be found in the work of Yoshioka, e.g., [30, Lemma 2.1]. Our computations below are based on similar techniques.

\section{Extremal rays from $\mathbb{P}^{1}$-bundles.}

EXAMPLE 4.2. When $X \simeq S^{[n]}, \delta^{\vee}$ is naturally an extremal ray of $S^{[n]}$. Consider the contraction to the symmetric product

$$
\begin{aligned}
\gamma: S^{[n]} & \rightarrow S^{n} / \mathfrak{S}_{n} \\
\Sigma & \mapsto \operatorname{supp}(\Sigma)
\end{aligned}
$$

assigning to each subscheme its support. Consider the open set $\Delta_{\circ}^{[n]} \subset \Delta^{[n]}$ corresponding to subschemes whose support consists of $n-1$ distinct points. The contraction $\gamma$ induces a morphism forgetting the nontrivial scheme structure:

$$
\begin{aligned}
& \gamma: \Delta_{\circ}^{[n]} \rightarrow\left(S^{[n-2]} \times S\right) \text { 。 } \\
& \Sigma \mapsto \operatorname{red}(\Sigma) \text {. }
\end{aligned}
$$

The fibers are isomorphic to $\mathbb{P}^{1}$ and have class $\delta^{\vee}$ by the formula

$$
\mathcal{O}_{S[n]}\left(\Delta^{[n]}\right) \mid \ell \simeq \mathcal{O}_{\mathbb{P}^{1}}(-2)
$$

As we've seen, $\left(\delta^{\vee}, \delta^{\vee}\right)=-1 / 2(n-1)$. 
EXAMPLE 4.3. Consider the case where $(S, f)$ is a general K3 surface of degree $2\left(r^{2}+r\right)$ for some positive integer $r$. Consider the moduli space

$$
M_{v}(S), \quad v=(r, f,(r+1) p)
$$

parametrizing simple sheaves $\mathcal{E}$ with invariants

$$
c_{1}(\mathcal{E})=f, r(\mathcal{E})=r, c_{2}(\mathcal{E})=r^{2}+r-1, \chi(\mathcal{E})=2 r+1 .
$$

The resulting isogenous K3 surface $M$ has degree $2\left(r^{2}+r\right)$ as well.

We apply the Fourier-Mukai functor to $S^{[2]}$ which is isomorphic to

$$
M_{w}(S), \quad w=(1,0,-p) .
$$

We compute

$$
\phi_{\mathcal{E}}(w)=\left((r+1)+f^{\prime}+r p^{\prime}\right)-\left(r+f^{\prime}+(r+1) p^{\prime}\right)=1-p^{\prime},
$$

i.e., $\phi_{\mathcal{E}}(w)$ has the invariants of ideals defining elements of $M^{[2]}$. This is symmetric in $S$ and $M$ and induces a birational map

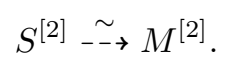

The diagonal in $M^{[2]}$ yields a divisor in $S^{[2]}$ ruled in $\mathbb{P}^{1}$ s over $M$.

We can interpret this divisor in $\mathrm{H}^{2}\left(S^{[2]}, \mathbb{Z}\right)$ : The class $\delta^{\prime}=2 f-(2 r+1) \delta$ satisfies

$$
\left(\delta^{\prime}, \delta^{\prime}\right)=-2,\left(\delta^{\prime}, \mathrm{H}^{2}\left(S^{[2]}, \mathbb{Z}\right)\right)=2 \mathbb{Z}
$$

so our conjecture predicts $2 \delta^{\prime}$ is ruled by $\mathbb{P}^{1}$ s over a $\mathrm{K} 3$ surface.

EXAmple 4.4. Here we give examples of $\mathbb{P}^{1}$-bundles over $S_{8 n-16}^{[n-2]} \times S_{2 n-4}$ in $S_{8 n-16}^{[n]}$. Let $\left(S_{8 n-16}, f_{8 n-16}\right)$ be a degree $(8 n-16) \mathrm{K} 3$ surface in $\mathbb{P}^{4 n-7}$. Consider the moduli space of rank-two simple sheaves over $S_{8 n-16}$ with Chern classes $c_{1}(\mathcal{E})=$ $f_{8 n-16}$ and $c_{2}(\mathcal{E})=(2 n-4) p$. These have invariants

$$
\chi(\mathcal{E})=2(n-1), \quad v=\left(2, f_{8 n-16}, 2 n-4\right) .
$$

The moduli space $M_{v}\left(S_{8 n-16}\right)$ is isomorphic to a K3 surface $S_{2 n-4}$ of degree $2 n-4$.

Let $\mathcal{E}$ denote the universal sheaf over $S_{8 n-16} \times M_{v}\left(S_{8 n-16}\right)$ and $\mathcal{I}$ the universal ideal sheaf over $S_{8 n-16} \times S_{8 n-16}^{[n-2]}$. Consider the Fourier-Mukai transform defined over

$$
M_{v}\left(S_{8 n-16}\right) \times S_{8 n-16} \times S_{8 n-16}^{[n-2]}
$$

by the rule

$$
\mathcal{F}=\mathbb{R} \pi_{13 *}\left(\pi_{12}^{*} \mathcal{E} \otimes_{\mathbb{L}} \pi_{23}^{*} \mathcal{I}\right) .
$$

For generic points $\mathcal{E}_{m} \in M_{v}\left(S_{8 n-16}\right)$ and $p_{1}+\ldots+p_{n-2} \in S_{8 n-16}^{[n-2]}$ we have

$$
\mathcal{F}_{\left(m, p_{1}+\ldots+p_{n-2}\right)}=\Gamma\left(S_{8 n-16}, \mathcal{E}_{m}\left(-p_{1}-\ldots-p_{n-2}\right)\right)
$$

which is two-dimensional as

$$
\chi\left(\mathcal{E}_{m} \otimes \mathcal{I}_{p_{1}+\ldots+p_{n-2}}\right)=\chi\left(\mathcal{E}_{m}\right)-2(n-2)=2 ;
$$


see $[31, \S 2]$ (esp. Lemma 2.6) for a general discussion of jumping behavior for dimensions of spaces of global sections over moduli spaces of sheaves.

Thus we obtain the diagram

$$
\begin{aligned}
& \mathbb{P}^{1} \hookrightarrow \quad \mathbb{P}(\mathcal{F}) \quad \stackrel{c_{2}}{\longrightarrow} S_{8 n-16}^{[n]} \\
& \alpha \downarrow \\
& M_{v}\left(S_{8 n-16}\right) \times S_{8 n-16}^{[n-2]}
\end{aligned}
$$

which gives the desired fibered subvariety.

EXAMPLE 4.5. We specialize this analysis to $n=4$, in which case we have Mukaiisogenous $\mathrm{K} 3$ surfaces $S_{16} \subset \mathbb{P}^{9}$ and $S_{4} \subset \mathbb{P}^{3}$. These have been extensively studied [15][25].

We claim that the fibers $\alpha$ map to smooth rational curves $\mathbb{P}^{1} \subset S_{8 n-16}^{[n]}$ with class

$$
R=f_{16}-10 \delta^{\vee}
$$

which satisfies $(R, R)=-2 / 3$.

Choose a generic sheaf $\mathcal{E}_{m}$ over $S_{16}$ corresponding to a point $m \in M_{v}\left(S_{16}\right) \simeq S_{4}$. Recall that $c_{1}\left(\mathcal{E}_{m}\right)=f_{16}, c_{2}\left(\mathcal{E}_{m}\right)=6$, and $\chi\left(\mathcal{E}_{m}\right)=6$. Since $\mathcal{E}_{m}$ is generically globally generated by six sections, we obtain a classifying map

$$
\begin{aligned}
& S_{16} \rightarrow \operatorname{Gr}(4,6) \\
& s \mapsto \Lambda_{s},
\end{aligned}
$$

uniquely defined up to projectivities. Choose $s_{1}+s_{2} \in S_{16}^{[2]}$ and corresponding codimension two linear subspaces

$$
\Lambda_{1}, \Lambda_{2} \in \mathbb{P}^{5}
$$

Let $\ell_{12} \subset \mathbb{P}^{5}$ denote the line where they intersect.

Let $H \subset S_{16}$ denote the hyperplane section defined by the Schubert class associated to $\ell_{12}$, i.e.,

$$
H=\left\{s \in S_{16}: \Lambda_{s} \cap \ell_{12} \neq \emptyset\right\}
$$

which contains $s_{1}$ and $s_{2}$ with multiplicity $>1$. Indeed, $\ell_{12}$ is contained in $\Lambda_{1}$ and $\Lambda_{2}$, not just incident to them. We conclude that $H$ has nodes at $s_{1}$ and $s_{2}$. Let $\tilde{H}$ denote the normalization of $H$, which is generically of genus $9-2=7$.

Consider the induced linear series

$$
\begin{aligned}
\varphi: \tilde{H} & \rightarrow \ell_{12} \\
s & \mapsto \Lambda_{s} \cap \ell_{12} .
\end{aligned}
$$

A Schubert-class computation

$$
\sigma_{2}=\sigma_{1}^{2}-\sigma_{11}
$$

gives

$$
\operatorname{deg}(\varphi)=\sigma_{2} \cdot S_{16}-2=16-10-2=4,
$$

taking into account that $s_{1}, s_{2}$ are basepoints. 
We conclude that $R$ corresponds to a $g_{4}^{1}$ on a genus-seven hyperplane section to $S_{16}$. Thus $R \cdot f_{16}=16$ and $R \cdot \delta^{\vee}=10$ by the Hurwitz formula. We deduce that

$$
R=f_{16}-10 \delta^{\vee} \in \mathrm{H}_{2}\left(S_{16}^{[4]}, \mathbb{Z}\right)
$$

and $(R, R)=-2 / 3$.

Example 4.6. Let $\left(S_{2(n-2)}, f\right)$ denote a polarized K3 surface. Consider the relative Picard scheme

$$
\operatorname{Pic}^{n}\left(S_{2(n-2)},|f|\right)=M_{v}\left(S_{2(n-2)}\right), \quad v=(0, f, 2) .
$$

In other words, these are degree $n$ invertible sheaves on hyperplane sections of $S_{2(n-2)}$, which have genus $n-1$. Consider the relative Hilbert scheme

$$
S_{2(n-2)}^{[n]}(|f|) \subset S_{2(n-2)}^{[n]}
$$

consisting of length $n$ subschemes $\Sigma \subset S_{2(n-2)}$ lying in hyperplane sections. The Abel-Jacobi map

$$
\alpha: S_{2(n-2)}^{[n]}(|f|) \rightarrow \operatorname{Pic}^{n}\left(S_{2(n-2)},|f|\right)
$$

is a $\mathbb{P}^{1}$-bundle. (Generally a degree $n$ line bundle on a genus $n-1$ curve has two sections by Riemann-Roch.) Thus we obtain the diagram

$$
\begin{aligned}
& \mathbb{P}^{1} \hookrightarrow S_{2(n-2)}^{[n]}(|f|) \quad \rightarrow \quad S_{2(n-2)}^{[n]} \\
& \alpha \downarrow \\
& M_{0, f, 2}\left(S_{2(n-2)}\right)
\end{aligned}
$$

Note that this moduli space is deformation equivalent to $T^{[n-1]}$ for $T$ a K3 surface (cf. [31, Thm. 0.1]). Indeed, specialize to the situation where $S_{2(n-2)}$ contains a line $\ell \simeq \mathbb{P}^{1}$, i.e., $f \cdot \ell=1$. There is an isomorphism

$$
\begin{aligned}
\operatorname{Pic}^{n}\left(S_{2(n-2)},|f|\right) & \rightarrow \operatorname{Pic}^{n-1}\left(S_{2(n-2)},|f|\right) \\
\mathcal{E} & \mapsto \mathcal{E} \otimes \mathcal{O}(-\ell)
\end{aligned}
$$

and the latter variety is birational to $S_{2(n-2)}^{[n-1]}$ by the cycle class map. Any two birational holomorphic symplectic manifolds are deformation equivalent [12, Theorem 4.6].

ExAmple 4.7. Let $S$ be a K3 surface containing a (-2)-curve $E \subset S$. Abusing notation, we also write $E$ for the class of the divisor

$$
\{Z: Z \cap E \neq \emptyset\} \subset S^{[n]} .
$$

This divisor admits an open subset isomorphic to

$$
(S \backslash E)^{[n-1]} \times \mathbb{P}^{1} .
$$

Let $R$ denote the class of a generic ruling; we have $R \cdot E=-2$ hence $R$ equals $E$ (via the inclusion $\mathrm{H}^{2}\left(S^{[n]}, \mathbb{Z}\right) \subset \mathrm{H}_{2}\left(S^{[n]}, \mathbb{Z}\right)$.)

The class $R$ is not extremal in the cone of curves of $S^{[n]}$; indeed $\Pi \simeq E^{[n]} \subset S^{[n]}$ is Lagrangian and the class of a line $\ell$ is the extremal class (see Example 4.11.) Nevertheless, we can deform $S^{[n]}$ so that $R$ remains algebraic but [ $\left.\ell\right]$ does not, and thus the rulings of (2) deform to rational curves in nearby fibers $[8,27]$. 
Extremal rays from $\mathbb{P}^{n-2}$-bundles. Let $S_{4 n-10} \subset \mathbb{P}^{2 n-4}$ denote a generic K3 surface of degree $4 n-10$ with polarization $f$. Let $n \geq 3$ and consider the moduli space of simple sheaves $\mathcal{E}$ on $S_{4 n-10}$ with invariants

$$
c_{1}(\mathcal{E})=f, c_{2}(\mathcal{E})=n, r(\mathcal{E})=2 .
$$

We can compute

$$
\chi(\mathcal{E})=n-1, \quad s(\mathcal{E})=\chi(\mathcal{E})-r(\mathcal{E})=n-3
$$

and the Mukai vector

$$
v=(2, f, n-3), \quad\langle v, v\rangle=(4 n-10)-2 \cdot 2 \cdot(n-3)=2 .
$$

Thus $M_{v}\left(S_{4 n-10}\right)$ has dimension four.

Let $\mathcal{E}$ be the universal bundle over $S_{4 n-10} \times M_{v}(S)$ and $\mathcal{F}=\left(\pi_{2}\right)_{*} \mathcal{E}$ which is a vector bundle of rank $n-1$. We have the following diagram

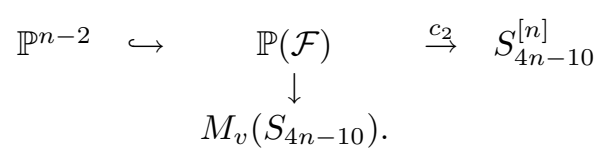

EXAMPLE 4.8. We specialize to the case where $n=4$, so the K3 surface

$$
S_{4 n-10}=S_{6} \subset \mathbb{P}^{4}
$$

has degree six. Here we consider rank-two simple sheaves with $c_{1}(\mathcal{E})=f, c_{2}(\mathcal{E})=4$, and $\chi(\mathcal{E})=3$. The classifying maps associated to such $\mathcal{E}$ are degree four rational maps

$$
\mu_{\mathcal{E}}: S_{6} \rightarrow \mathbb{P}^{2}
$$

with the line class on $\mathbb{P}^{2}$ pulling back to the polarization on $S_{6}$. Let $Q$ denote the rank-two universal quotient bundle on $\mathbb{P}^{2}$ so that $\mu^{*} Q=\mathcal{E}$ after extending over the indeterminacy.

We construct the classifying maps explicitly: Fix an ordinary secant line $L\left(s_{1}+s_{2}\right)$ of $S_{6} \subset \mathbb{P}^{4}$ meeting $S_{6}$ at $s_{1}$ and $s_{2}$. Projection induces a rational map of degree four

$$
\pi_{L\left(s_{1}+s_{2}\right)}: S_{6} \rightarrow \mathbb{P}^{2}
$$

resolved by blowing up $s_{1}$ and $s_{2}$. The association

$$
\begin{array}{lll}
\phi: S_{6}^{[2]} & -\rightarrow & M_{(2, f, 1)}\left(S_{6}\right) \\
s_{1}+s_{2} & \mapsto & \pi_{L\left(s_{1}+s_{2}\right)}^{*} Q
\end{array}
$$

is birational. Thus $S_{6}^{[2]}$ is deformation equivalent to the compact moduli space associated with $M_{(2, f, 1)}\left(S_{6}\right)$. Note that the map $\phi$ is not regular; it has indeterminacy along the three-secant lines of $S_{6}$, parametrized by the maximal isotropic subspaces of the quadric hypersurface containing $S_{6}$, which is isomorphic to $\mathbb{P}^{3}$. 
Extremal rays from $\mathbb{P}^{n-1}$-bundles. Let $S_{4 n-8} \subset \mathbb{P}^{2 n-3}$ be a general K3 surface of degree $4 n-8$ with polarization $f$; assume $n \geq 3$. Consider the moduli space of simple sheaves on $S_{4 n-8}$ with the following invariants:

$$
c_{1}(\mathcal{E})=f, \quad c_{2}(\mathcal{E})=n, \quad r(\mathcal{E})=2 .
$$

We get the auxiliary invariants

$$
\chi(\mathcal{E})=n, \quad s(\mathcal{E})=\chi(\mathcal{E})-r(\mathcal{E})=n-2
$$

which determine a Mukai vector

$$
v=\left(r(\mathcal{E}), c_{1}(\mathcal{E}), s(\mathcal{E})\right), \quad\langle v, v\rangle=c_{1}(\mathcal{E})^{2}-2 r(\mathcal{E}) s(\mathcal{E})=0 .
$$

Let $M_{v}\left(S_{4 n-8}\right)$ denote the corresponding moduli space, which is isomorphic to a K3 surface $T$. Let $\mathcal{E}$ be the universal bundle over $S_{4 n-8} \times M_{v}(S)$. Let $\mathcal{F}=\left(\pi_{2}\right)_{*} \mathcal{E}$ which is a vector space of dimension four. We have the following diagram

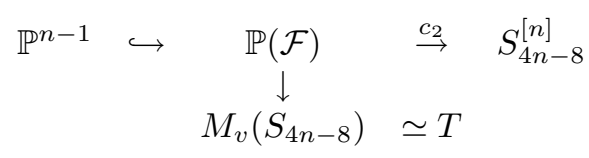

EXAmple 4.9. Consider the special case $n=3$, i.e., rank two simple sheaves $\mathcal{E}$ on a quartic surface $S_{4}$ with $c_{1}(\mathcal{E})=f, c_{2}(\mathcal{E})=3$. We expect the classifying maps of these to be rational maps

$$
\mu: S_{4} \rightarrow \operatorname{Gr}(1,3) \simeq \mathbb{P}^{2},
$$

well-defined where $\mathcal{E}$ is free and globally generated. The map $\mu$ has degree three and $\mathcal{E}$ coincides with an extension of $\mu^{*} Q$, where $Q$ is the tautological quotient sheaf on $\mathbb{P}^{2}$. (Note that $\mu^{*} Q$ is well-defined away from a finite subset of $S_{4}$, and thus admits unique extension to a torsion-free sheaf with the specified invariants.) Thus $\mu$ is the projection from some point $p \in S_{4}$; the corresponding bundle is denoted $\mathcal{E}_{p}$.

What are the loci where sections of $\mathcal{E}_{p}$ vanish? We assume $S_{4}$ does not contain a line. Sections of $Q$ vanish at points of $\mathbb{P}^{2}$. For each line

$$
p \in L_{t} \subset \mathbb{P}^{3}, \quad t \in \mathbb{P}^{2},
$$

we have

$$
L_{t} \cap S_{4}=\left\{p, s_{1}, s_{2}, s_{3}\right\} .
$$

Scheme-theoretically, we take the residual scheme to $p$ in $L_{t} \cap S_{4}$, which is a welldefined length three subscheme $\Sigma_{t} \subset S_{4}$. Thus we get a morphism

$$
\begin{aligned}
\mathbb{P}^{2} & \rightarrow S_{4}^{[3]} \\
t & \mapsto \Sigma_{t} .
\end{aligned}
$$

EXAMPLE 4.10. In the special case $n=4$ we recover an example of Mukai [22, Example 0.9]. In this situation $M_{v}\left(S_{8}\right) \simeq S_{2}$, a degree two K3 surface. Our diagram takes the form

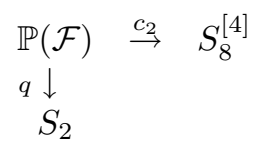

where $q$ is a $\mathbb{P}^{3}$ bundle. 
Extremal rays from Lagrangian subspaces. Here is a simple-sheaf construction of a Lagrangian $\mathbb{P}^{n} \subset S^{[n]}$ for special K3 surfaces $S$.

EXAMPLE 4.11. Let $S$ be a K3 surface containing a nonsingular rational curve $E \subset S$, which induces

$$
\Pi \simeq E^{[n]} \subset S^{[n]}
$$

Lines in $\Pi$ correspond to pencils of binary forms of degree $n$, e.g., families of subscheme

$$
\ell=\left\{e_{1}+\ldots+e_{n}: e_{1}, \ldots, e_{n-1} \text { fixed }, e_{n} \in E \text { varying }\right\} .
$$

Since the discriminant of such a form has degree $2(n-1)$ in the coefficients, we find

$$
\Delta^{[n]} \cdot \ell=2(n-1) .
$$

Let $E$ denote the divisor in $S^{[n]}$ parametrizing subschemes with some support on $E$. Deforming the $e_{1}, \ldots, e_{n-1}$ to generic points of $S$, we find that $\ell \cdot E=-2$ and

$$
\ell=E-(n-1) \delta^{\vee} .
$$

EXAmPle 4.12. Let $\left(S_{10}, f\right)$ be a polarized K3 surface of degree ten. We can express

$$
S_{10}=\Lambda \cap Q \cap \operatorname{Gr}(2,5)
$$

where $Q$ is a quadric hypersurface and $\Lambda$ is a linear subspace of codimension three. The variety of lines on $\Lambda \cap \operatorname{Gr}(2,5)$ is isomorphic to $\mathbb{P}^{2}$; each such line is 2-secant to $S_{10}$. This gives us an inclusion

$$
\mathbb{P}^{2} \hookrightarrow S_{10}^{[2]} .
$$

Here is a vector bundle interpretation: Let $\mathcal{E}$ denote the rigid vector bundle represented by the moduli space $M_{2, f, 3}\left(S_{10}\right)$, which is the restriction of the ranktwo tautological quotient bundle of $\operatorname{Gr}(2,5)$ to $S_{10}$ via the embedding given above. A generic point $s \in S_{10}$ determines a codimension two subspace $K_{s} \subset \Gamma\left(S_{10}, \mathcal{E}\right)$. Consider the locus

$$
\Pi:=\left\{s_{1}+s_{2} \in S_{10}^{[2]}: \operatorname{dim} K_{s_{1}} \cap K_{s_{2}} \geq 2\right\} .
$$

This coincides with the plane constructed in the previous paragraph.

Let $S_{4 n-6} \subset \mathbb{P}^{2 n-2}$ be a general degree $(4 n-6) \mathrm{K} 3$ surface with hyperplane class $f$. (When $n=2$ the map to $\mathbb{P}^{2}$ is two-to-one.) Consider the moduli space of simple sheaves $\mathcal{E}$ on $S_{4 n-6}$ with the following invariants:

$$
c_{1}(\mathcal{E})=f, \quad c_{2}(\mathcal{E})=2 n, \quad r(\mathcal{E})=2 .
$$

We get the auxiliary invariants

$$
\chi(\mathcal{E})=n+1, \quad s(\mathcal{E})=\chi(\mathcal{E})-r(\mathcal{E})=n-1
$$


which determine a Mukai vector

$$
v=\left(r(\mathcal{E}), c_{1}(\mathcal{E}), s(\mathcal{E})\right), \quad\langle v, v\rangle=c_{1}(\mathcal{E})^{2}-2 r(\mathcal{E}) s(\mathcal{E})=-2 .
$$

Let $M_{v}\left(S_{4 n-6}\right)=$ \{point denote the corresponding moduli space and $\mathcal{E}$ the universal sheaf over $S_{4 n-6} \times M_{v}(S)$. Let $\mathcal{F}=\left(\pi_{2}\right)_{*} \mathcal{E}$ which is a vector space of dimension $n+1$. We have the following diagram

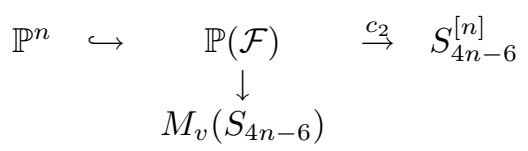

which induces our Lagrangian $\mathbb{P}^{n}$.

EXAMPLE 4.13. We consider the special case where $n=3$, which goes back to Mukai [23]. Here we have a degree six K3 surface $S_{6}$ which can generically be expressed as a complete intersection of a smooth quadric and a cubic in $\mathbb{P}^{4}$ :

$$
S_{6}=W_{2} \cap W_{3} .
$$

We construct the rigid sheaf explicitly: Express $W_{2}$ as a hyperplane section of a smooth quadric fourfolds $W_{2}^{\prime}$ and fix an isomorphism $W_{2}^{\prime} \simeq \operatorname{Gr}(2,4)$ to the Grassmannian. Let $Q \rightarrow \operatorname{Gr}(2,4)$ denote the universal quotient bundle, which is globally generated by four sections. Then $\mathcal{E}$ is the restriction of $Q$ to $S_{6}$.

The zero-sections of $Q$ trace out maximal isotropic subspaces of $W_{2}^{\prime}$, which restrict to maximal isotropic subspaces of $W_{2}$, i.e., a family of lines $\left\{L_{t}\right\}$ parametrized by $\mathbb{P}^{3}$. The intersections $L_{t} \cap S_{6}$ are length three subschemes of $S_{6}$, at least assuming $S_{6}$ does not contain a line. Therefore, we obtain a morphism

$$
\begin{aligned}
\mathbb{P}^{3} & \rightarrow S_{6}^{[3]} \\
t & \mapsto L_{t} \cap S_{6} .
\end{aligned}
$$

\section{Data for generalized Kummer varieties.}

\begin{tabular}{|c|c|l|l|}
\hline$(R, R)$ & $(\rho, \rho)$ & Geometry & Example \\
\hline$-\frac{1}{6}$ & -6 & Diagonal $\mathbb{P}^{1}$-bundle over $A$ & see introduction \\
$-\frac{2}{3}$ & -6 & $\mathbb{P}^{1}$-bundle over $A$ & 6.1 \\
$-\frac{3}{2}$ & -6 & $\mathbb{P}^{2}$ (Lagrangian) & 6.3 \\
\hline
\end{tabular}

TABLE K2. $X \sim K_{2}(A)$ 


\begin{tabular}{|c|c|l|l|}
\hline$(R, R)$ & $(\rho, \rho)$ & Geometry & Example \\
\hline$-\frac{1}{2(n+1)}$ & $-2(n+1)$ & Diagonal $\mathbb{P}^{1}$-bundle over $A \times A^{[n-2]}$ & see $\S 1$ \\
$-\frac{2}{n+1}$ & $-2(n+1)$ & $\begin{array}{l}\mathbb{P}^{1} \text {-bundle over } \\
\text { holomorphic symplectic manifold }\end{array}$ & 6.1 \\
$\vdots$ & $\vdots$ & $\vdots$ & $\vdots$ \\
$-\frac{n+1}{2}$ & $-2(n+1)$ & $\mathbb{P}^{n}$ (Lagrangian) & 6.3 \\
\hline
\end{tabular}

Tentative Table Kn. $X \sim K_{n}(A)$

\section{Examples of extremal rays for generalized Kummer varieties.}

Extremal rays from $\mathbb{P}^{1}$-bundles. ExAMPLE 6.1. Let $C$ be a smooth projective curve of genus two and $(A, \Theta)$ its Jacobian. For simplicity, we assume that the NéronSeveri group of $A$ is generated by $\Theta$. Consider $K_{2}(A)$ with

$$
\operatorname{Pic}\left(K_{2}(A)\right)=\mathbb{Z} \Theta \oplus_{\perp} \mathbb{Z} e, \quad(\Theta, \Theta)=2,(e, e)=-6 .
$$

Each degree three line bundle $L$ on $C$ has $h^{0}(C, L)=2$, and is globally generated unless $L \simeq \omega_{C}(p)$ for some point $p \in C$. We can therefore consider the subvariety

$$
F^{\prime}=\left\{Z: Z \subset A \text { has length three, } Z \subset \tau_{a}(C) \text { for some } a \in A\right\} \subset A^{[3]},
$$

where $\tau_{a}$ is translation by $a$. Note that $F^{\prime}$ is a $\mathbb{P}^{1}$-bundle over the degree three component of the relative Picard scheme of the collection of translates of $C$ in $A$. Fixing a reference $j: C \hookrightarrow A$ induces a morphism of Albanese varieties $j_{*}: \mathrm{Alb}_{3}(C) \rightarrow$ $\operatorname{Alb}_{3}(A) \simeq A$, where the last isomorphism is translation by $3 \times(0)$. Since $A=$ $\operatorname{Alb}_{0}(C)=\operatorname{Alb}_{0}(A), j^{*}$ is compatible with the right actions of $A$. On the other hand,

$$
\left(\tau_{a} \circ j\right)_{*}=\tau_{3 a *} \circ j_{*} .
$$

Restricting to the Kummer subvariety

$$
F=F^{\prime} \cap K_{2}(A)
$$

yields a $\mathbb{P}^{1}$-bundle over

$$
\{(a, b) \in A \times A: 3 a+b=0\} \simeq A .
$$

Let $R$ denote the class of a generic fiber $\ell$ of this bundle. We know that $\ell \cdot \Theta=2$ and $\ell \cdot e=4$, because any degree three morphism $C \rightarrow \mathbb{P}^{1}$ has eight ramification points, counted with multiplicities. It follows that

$$
R=\Theta-\frac{2}{3} e
$$

which satisfies

$$
(R, R)=-\frac{2}{3} .
$$


Let $\rho=3 R$ denote the smallest positive multiple of $R$ contained in $\mathrm{H}^{2}\left(K_{2}(A), \mathbb{Z}\right)$; we have

$$
(\rho, \rho)=-6, \quad\left(\rho, \mathrm{H}^{2}\left(K_{2}(A), \mathbb{Z}\right)\right)=3 \mathbb{Z}
$$

ExAmple 6.2. Let $(A, \Theta)$ be an abelian surface with $(1, \mathrm{~g})$ polarization $\Theta$ and Néron-Severi group generated by $\Theta$. Consider $K_{\mathrm{g}}(A)$ with

$$
\operatorname{Pic}\left(K_{\mathrm{g}}(A)\right)=\mathbb{Z} \Theta \oplus_{\perp} \mathbb{Z} e, \quad(\Theta, \Theta)=2 \mathrm{~g}-2,(e, e)=-2(\mathrm{~g}+1) .
$$

Consider degree $(\mathrm{g}+1)$ line bundles $L$ on curves $C$ homologically equivalent to $\Theta$. We have $h^{0}(C, L) \geq 2$ with equality for general $C$ and $L$. We have the locus

$$
F^{\prime}=\left\{Z: Z \subset A \text { has length } \mathrm{g}+1, Z \subset \tau_{a}(C) \text { for some } a \in A, C \in|\Theta|\right\}
$$

in $A^{[\mathrm{g}+1]}$. Accounting for the fibers of $C^{[\mathrm{g}+1]} \rightarrow J^{\mathrm{g}+1}(C)$, translations of $C$, and linear equivalences within each translated divisor class, we find

$$
\operatorname{dim}\left(F^{\prime}\right)=1+\mathrm{g}+2+\mathrm{g}-2=2 \mathrm{~g}+1 .
$$

In particular, $F^{\prime}$ is a divisor fibered as follows:

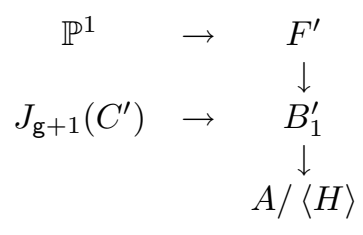

where

- the first fibration reflects $g_{\mathrm{g}+1}^{1}$ 's on deformations $C^{\prime}$ of $C$;

- the second corresponds to the degree $(\mathrm{g}+1)$ relative Jacobian of the $C^{\prime}$; and

- $H$ is the torsion subgroup preserving $\Theta$.

Let $F=F^{\prime} \cap K_{\mathrm{g}}(A)$, which is also fibered in $\mathbb{P}^{1}$ 's over a $(2 \mathrm{~g}-2)$-dimensional irreducible holomorphic symplectic manifold (cf. [30, Thm. 1.4], [32, Thm. 0.1]). Let $R$ be the class of a ruling which, which meets the diagonal divisor $2 e$ in $4 \mathrm{~g}$ points and $\Theta$ in $2 \mathrm{~g}-2$ points. Thus

$$
R=\Theta-\frac{\mathrm{g}}{(\mathrm{g}+1)} e
$$

with

$$
(R, R)=2 \mathrm{~g}-2-\frac{2 \mathrm{~g}^{2}}{(\mathrm{~g}+1)}=-\frac{2}{\mathrm{~g}+1} .
$$

Let $\rho=(\mathrm{g}+1) R$, the smallest positive multiple in $\mathrm{H}^{2}\left(K_{\mathrm{g}}(A), \mathbb{Z}\right)$; then $(\rho, \rho)=$ $-2(\mathrm{~g}+1)$. Again, this is equal to $(e, e)$. 


\section{Extremal rays from Lagrangian subspaces.}

Example 6.3. Let $\left(E_{1}, p_{1}\right)$ and $\left(E_{2}, p_{2}\right)$ be elliptic curves. Let $A=E_{1} \times E_{2}$ and $X=K_{n}(A)$. Consider the projective space

$$
P=\left\{D \times p_{2}: \text { where } D \in\left|(n+1) p_{1}\right|\right\} \simeq \mathbb{P}^{n} .
$$

Let $\ell \subset P$ be a line. Then its class

$$
R=[\ell] \in \mathrm{H}_{2}(X, \mathbb{Z}) \subset \mathrm{H}^{2}(X, \mathbb{Z}) \otimes_{\mathbb{Z}} \mathbb{Q}
$$

can be expressed

$$
R=E_{1}-\frac{1}{2} e
$$

In particular,

$$
(R, R)=-(n+1) / 2, \quad \rho:=2 R \in \mathrm{H}^{2}(X, \mathbb{Z}),(\rho, \rho)=-2(n+1) .
$$

This again is equal to $(e, e)$.

Indeed, since $\operatorname{Pic}\left(K_{n}(A)\right)$ is generated by the classes of $E_{1}, E_{2}$, and $e$, so it remains to solve for the coefficients of

$$
R=c_{1} E_{1}+c_{2} E_{2}+c_{0} e .
$$

Interpreting $\ell$ is a pencil of degree $n+1$ on $E_{1}$, we have

$$
\ell \cdot E_{1}=0, \quad \ell \cdot E_{2}=1, \quad \ell \cdot e=(n+1) .
$$

\section{REFERENCES}

[1] A. Beauville, Variétés Kähleriennes dont la première classe de Chern est nulle, J. Differential Geom., 18:4 (1983), pp. 755-782.

[2] C. Birkar, P. Cascini, Chr. D. Hacon, and J. McKernan, Existence of minimal models for varieties of log general type, J. Amer. Math. Soc., 23 (2010), pp. 405-468.

[3] S. Boucksom, Divisorial Zariski decompositions on compact complex manifolds, Ann. Sci. École Norm. Sup. (4), 37:1 (2004), pp. 45-76.

[4] K. Cho, Y. MiYaoka, And N. I. Shepherd-BARron, Characterizations of projective space and applications to complex symplectic manifolds, in "Higher dimensional birational geometry (Kyoto, 1997)", volume 35 of Adv. Stud. Pure Math., pp. 1-88. Math. Soc. Japan, Tokyo, 2002.

[5] H. Clemens, J. Kollár, And Sh. Mori, Higher-dimensional complex geometry, Astérisque, 166 (1988).

[6] S. DRuEL, Quelques remarques sur la décomposition de Zariski divisorielle sur les variétés dont la premierère classe de Chern est nulle, 2009. arXiv:0902.1078v2.

[7] V. Gritsenko, K. Hulek, and G. K. Sankaran, Moduli spaces of irreducible symplectic manifolds, arXiv:0802.2078, to appear in "Compositio Mathematica".

[8] B. Hassett and Y. Tschinkel, Rational curves on holomorphic symplectic fourfolds, Geom. Funct. Anal., 11:6 (2001), pp. 1201-1228.

[9] B. Hassett And Y. Tschinkel, Moving and ample cones of holomorphic symplectic fourfolds, Geom. Funct. Anal., 19:4 (2009), pp. 1065-1080.

[10] B. Hassett And Y. TsChinkel, Flops on holomorphic symplectic fourfolds and determinantal cubic hypersurfaces, J. Inst. Math. Jussieu, 9:1 (2010), pp. 125-153. 
[11] B. Hassett And Y. Tschinkel, Hodge theory and Lagrangian planes on generalized Kummer fourfolds, 2010. arXiv:1004.0046.

[12] D. Huybrechts, Compact hyper-Kähler manifolds: basic results, Invent. Math., 135:1 (1999), pp. 63-113.

[13] D. Huybrechts, The Kähler cone of a compact hyperkähler manifold, Math. Ann., 326:3 (2003), pp. 499-513.

[14] D. HuYBRECHTS, Fourier-Mukai transforms in algebraic geometry, Oxford Mathematical Monographs, The Clarendon Press Oxford University Press, Oxford, 2006.

[15] A. Iliev AND K. RANestad, The abelian fibration on the Hilbert cube of a K3 surface of genus 9, Internat. J. Math., 18:1 (2007), pp. 1-26.

[16] D. Kaledin, Symplectic singularities from the Poisson point of view, J. Reine Angew. Math., 600 (2006), pp. 135-156.

[17] S. L. Kleiman, Toward a numerical theory of ampleness, Ann. of Math. (2), 84 (1966), pp. 293-344.

[18] E. Looijenga And C. Peters, Torelli theorems for Kähler K3 surfaces, Compositio Math., 42:2 (1980/81), pp. 145-186.

[19] E. MARKMAN, Integral constraints on the monodromy group of the hyperkähler resolution of a symmetric product of a K3 surface, 2006. arXiv:math/0601304.

[20] E. Markman, On the monodromy of moduli spaces of sheaves on K3 surfaces, J. Algebraic Geom., 17:1 (2008), pp. 29-99.

[21] E. Markman, Prime exceptional divisors on holomorphic symplectic varieties, 2009. arXiv:0912.4981.

[22] Sh. MukaI, Symplectic structure of the moduli space of sheaves on an abelian or K3 surface, Invent. Math., 77:1 (1984), pp. 101-116.

[23] Sh. MukaI, Moduli of vector bundles on K3 surfaces and symplectic manifolds, Sūgaku, 39:3 (1987), pp. 216-235. Sugaku Expositions, 1:2 (1988), pp. 139-174.

[24] Sh. MukaI, On the moduli space of bundles on K3 surfaces. I, in "Vector bundles on algebraic varieties (Bombay, 1984)", volume 11 of Tata Inst. Fund. Res. Stud. Math., pp. 341-413. Tata Inst. Fund. Res., Bombay, 1987.

[25] Sh. Mukai, Curves, K3 surfaces and Fano 3-folds of genus $\leq 10$, in "Algebraic geometry and commutative algebra, Vol. I", pp. 357-377, Kinokuniya, Tokyo, 1988.

[26] Y. Namikawa, Deformation theory of singular symplectic n-folds, Math. Ann., 319:3 (2001), pp. 597-623.

[27] Z. RAN, Hodge theory and deformations of maps, Compositio Math., 97:3 (1995), pp. 309-328.

[28] J. SAwON, Abelian fibred holomorphic symplectic manifolds, Turkish J. Math., 27:1 (2003), pp. 197-230.

[29] J. WierzBA, Contractions of symplectic varieties, J. Algebraic Geom., 12:3 (2003), pp. 507534.

[30] K. YoshiokA, Some examples of isomorphisms induced by Fourier-Mukai functors, 1999. arXiv:math/9902105v1.

[31] K. Yoshioka, Some examples of Mukai's reflections on K3 surfaces, J. Reine Angew. Math., 515 (1999), pp. 97-123.

[32] K. Yoshioka, Moduli spaces of stable sheaves on abelian surfaces, Math. Ann., 321:4 (2001), pp. 817-884. 